\title{
VIDEO-ASSISTED THORACOSCOPIC BILATERAL SYMPATHECTOMY FOR PRIMARY HYPERHIDROSIS IN ZAGAZIG UNIVERSITY HOSPITALS
}

\author{
Yehia Zakaria Atwaa, Mohamed Rashad Mohamed, Emad Mohamed Salah \\ and Rasha Shaaban Abdel Maksoud. \\ Department Of General Surgery, Zagazig University Hospitals, faculty of medicine, Zagazig, Egypt.
}

\begin{abstract}
Background:. Hyperhidrosis is the condition characterized by abnormally increased sweating/perspiration in excess of that required for regulation of body temperature. Hyperhydrosis can be primary or secondary. Primary hyperhidrosis is idiopathic in nature and the most affected regions are palms, axillae and feet. Palmar and axillo-palmar hyperhidrosis is a disabling disorder. It usually has negative impact on the patient's social and professional life. The incidence of primary hyperhydrosis is estimated to be around 0.6 to $1 \%$. Management strategies to hyperhidrosis may be classified as nonsurgical or surgical Treatment. Thoracoscopic sympathectomy is considered the most effective and durable treatment for patients suffering from moderate to severe hyperhidrosis with good short-term results and minimal postoperative complications Aim of the work: The aim of the work is to evaluate and compare the immediate and long-term outcomes of video-assisted thoracoscopic sympathectomy in the treatment of primary hyperhidrosis.Patients and Methods: From Jul. 2012 to Jun. 2017, a prospective study was conducted on fifty six patients, admitted to surgery Departments, zagazig university hospitals scheduled for bilateral thoracoscopic sympathectomy,for the treatment of primary hyperhydrosis involving the palms of both hands in all patients with or without involvement of the axillae.All the patients were considered in grades 3 or 4 of the HDSS in which their lifestyle and occupation were markedly affected.The sample included38 (67.9\%) males and 18 (32.1\%) female with mean age of 27.03 \pm 4.89 . Statistical analysis: SPSS version 20. Results: In total number of 56 patients with primary hyperhidrosis who passed bilateral thoracoscopic sympathectomy, it was found that all patients had immediate stoppage of palmar hyperhidrosis. The mean hospital stay was1.08 \pm 0.28 . Good Satisfaction had occurred in 51 cases $(91.1 \%)$ The most common postoperative troublesome was pleuritic chest pain in 18 cases(32.1\%). Compensatory sweating had occurred in 4 cases(7.1\%). 4 cases had developed postoperative pneumothorax. Conclusion:Video-assisted thoracoscopic bilateral sympathectomy is a rapid, reliable and safe technique. It presents important advantages over the staged procedures because it can be done in a single surgical procedure, requires placement of only two thoracic ports, avoiding the third port. The transaxillary bilateral approach provides as good if not better results and can certainly be done with similar or perhaps less morbidity. Furthermore, the bilateral approach reduces the time of patient recovery and reduces healthcare costs.

Key wards:Primary Hyperhydrosis, Thoracoscopic Sympathectomy and Compensatory Sweating.

Corresponding author: drsurgrasha@gmail.com
\end{abstract}

\section{INTRODUCTION}

$\mathrm{H}$ yperhidrosis is the condition characterized by abnormally increased sweating in excess of that required for regulation of body temperature. It is associated with a significant quality of life burden from a psychological, emotional, and social perspective. As such, it has been referred to as the 'silent handicap ${ }^{(\mathbf{1})}$.

The incidence of primary hyperhydrosis is generally estimated to be around 0.6 to $1 \%$. The condition starts in childhood but worsens around the time of puberty $^{(2)}$.. Sweat glands in patients with hyperhidrosis are identical to normal in number and histologically appearance. Hyperhidrosis caused by hyperfunction, which may be due to excessive sympathetic tone. Basal sweat production is above normal,and there is an exaggerated response to emotional or physical stress, although thermoregulation is normal ${ }^{(3)}$.
Although hyperhydrosis is not life threatening , it can be extremely disabling.. The stress associated with the sweating frequently leads to additional anxiety and a subsequent increase in sweating, which only exacerbates the condition ${ }^{(4)}$. Hyperhidrosis can either be generalized, or localized.. When excessive sweating is localized (e.g. palms, soles, face, underarms, scalp) it is referred to as primary hyperhidrosis or focal hyperhidrosis ${ }^{(5)}$. Excessive sweating involving the whole body is termed generalized hyperhidrosis or secondary hyperhidrosis. It is usually the result of some other, underlying condition such as Hyperthyroidism,Endocrine, Hyperpituitarism, Diabetes mellitus, Pregnancy and emotional factors ${ }^{(6)}$.

Many lines of treatment are available such as topical medications, iontopheresis, systemic medications, botulinum toxin type A (BTXA),Percutaneous
sympathicolysis and surgery ${ }^{(7)}$. Patients 
should generally start with the least invasive form of therapy and progress to more complicated therapies when other therapies fail $^{(8)}$.

Severe hyperhydrosis and cases where other treatments fail (Grade 3,4)' has been treated with thoracoscopic sympathectomy. Different approaches and techniques are available for thoracoscopic sympathectomy. bilateral simultaneous approach in supine position is considered one of the best choices, with good short-term results and minimal postoperative complications ${ }^{(9)}$.

This paper describes our experience with this technique and shows the postoperative degree of satisfaction and quality of life for our patients.

\section{AIM OF THE WORK}

The aim of the work is to evaluate and compare the immediate and long-term outcomes of video-assisted thoracoscopic sympathectomy in the treatment of primary hyperhidrosis.

\section{PATIENTS AND METHODS}

From Jul. 2012 to Jun. 2017, An approval of the ethical board of Zagazig University Hospitals and after obtaining a full informed written consent from each patient, a prospective study was conducted on fifty six patients admitted to surgery Departments, zagazig university hospitals scheduled for bilateral thoracoscopic sympathectomy, for the treatment of primary hyperhydrosis involving the palms of both hands in all patients with or without involvement of the axillae.All the patients were considered in grades 3 or 4 of the HDSS in which their lifestyle and occupation were markedly affected.The sample included38 (67.9\%) males and 18 $(32.1 \%)$ female with mean age of $27.03 \pm 4.89$. All patients were subjected to:

\section{I- Preoperative evaluation:}

1- Full history taking including age, occupation, history of hyperhidrosis.

2- Complete general examination.

3- Complete neurological examination.

4- Local examination of the hand: to define

- The rate of sweat production, the specific areas involved, the effect of the condition on the patient's quality of life, and the impairment of daily activities.
- $\quad$ Exact site and size of hyperhidrotic area.

- Degree of primary palmar hyperhidrosis by clinical methods.

5- Preoperative chest radiographs to exclude patients with preexisting pleural and/or pulmonary pathology.

6- Complete blood picture, renal and liver functions to determine fitness of patients and to exclude local or remote infection source.

7- Thyroid Function Panel, serum glucose levels, uric acid, and urine catecholamine level was done to rule out secondary hyperhidrosis.

\section{II- surgical intervention:}

In general,the operation is performed in supine or semifowler position with a pad placed under the vertebral column under general anesthesia using a double lumen endotracheal tube. The incision should be minimal due to the trocar size. double port with $5 \mathrm{~mm}$ trocar are used. The first incision is made in the fourth submammary intercostal space just below the pectoral muscle as an access route for camera, and a second incision is made at third midaxillary intercostal space to introduce surgical instruments. $5 \mathrm{~mm}$ trocars are employed, during brief disconnection of the endotracheal tube to deflate the lung when the pleural Cavity is entered to avoid damaging the lung parenchyma and for adequate visualization of the surgical field. Prior to the insertion of the camera, 8-10 $\mathrm{mmHg} 2 \mathrm{~L} / \mathrm{min}$ carbon dioxide insufflation can be performed in collapsing the lung and assisting visibility. Also semifowler position allows gravity to help pull the upper lobes out of the field of dissection.

The first rib is most often not visible. The rib heads from $\mathrm{T} 2$ to $\mathrm{T} 4$ are easily identifiable through the parietal pleura and are important landmarks during the thoracoscopic sympathectomy surgery and the second rib constitutes the superior boundary so, it is typically the first visible rib in thoracoscopic sympathectomy. The sympathetic chain courses superficial to the segmental and intercostal vessels. The stellate ganglion lies within the first intercostal space cephalid. the second rib head it is generally found underneath a fat pad at the superior limit of 
dissection. The sympathetic chain is easily seen as it is a slightly raised, longitudinal structure running parallel to the spine and coursing over the rib heads. Make a longitudinal incision in the parietal mediastinal pleura following the medial border of the sympathetic chain down to the fourth or fifth rib using discector. L-shaped Hook are then inserted under the mediastinal pleura to lift the sympathetic chain like a tent and to divide it caudad to cephalad with electrocauterty and once the upper thorax is reached, cautery should no longer be used to avoid diffusion of the current towards the cervicothoracic ganglion. The exposed sympathetic chain and associated $\mathrm{T} 3$ (T4) ganglion is isolated, the upper part of the chain is divided using scissors without cautery at the level of T2 which is the base of cervicothoracic ganglion, then the trunk of the freed nerve can be extracted via one of the trocars and removed from the thoracic cavity. The cervicothoracic (stellate) ganglion must be preserved. It is generally found underneath apad of fat at the superior limit of the dissection.

The T2 ganglion is inspected to identify the accessory Nerve of Kuntz which is frequently visible on the contour of the second rib, Although this anatomic variant is present in only approximately $10 \%$ of patients, it should be divided (if present) by cautious and brief cauterization and other accessory rami are not always visible so they are divided by cauterization of the periostem of the third and fourth ribs over a length of 3 to $4 \mathrm{~cm}$ starting from the neck of the ribs to avoid a failure of sympathectomy and to ensure complete resolution of the hyperhidrosis. At the end of the procedure, mild bleeding or oozing is common so it is preferable to achieve hemostasis by simple tamponade rather than by electrocautery. To do so a small gauze pad is first rolled up, introduced via the $5 \mathrm{~mm}$ trocar and genetly spread over the resection bed(the thoracoscope is withdrawn temporarly to allow placement of this gauze). The procedure is completed only after the operative field has been thoroughly evaluated and gas was then disconnected, the pleural cavity deflated and the $5 \mathrm{~mm}$ trocar was first removed under vision. At this stage, the anesthetist was asked to unclamp the ipsilateral lumen of the ETT and to hyperinflate the lung. With complete inflation of the lung the endoscopic trocar was removed and suture closed the trocar sites. The same procedure is applied to the contralateral side

\section{. III- Histopathology:}

All operative specimens were evaluated by histopathological examination. Specimens were processed in automated tissue processing units and stained with routine haematoxylin and eosin.

\section{IV-Postoperative care and follow up:}

For early and late complications. Follow up was done clinically, Data were collected by review of medical charts, telephone and interviews.for at least six months postoperatively.

\section{Statistical Analysis:}

Standard methods using SPSS version 20 for windows were used, Student t-test, Chi-square were used in appropriate situation. $P$. value $\leq 0.05$ was considered the level of significance.

\section{RESULTS}

Fifty Six patients with bilateral primary hyperhidrosis were enrolled in this study, they included $38(67.9 \%)$ males and $18(32.1 \%)$ females( Table:1) with mean age of $27.03 \pm 4.89$ years(Table:2).

All patients had immediate cessation of palmar hyperhidrosis. The mean postoperative stay was $1.08 \pm 0.28$ (range1-2) days, with a mode of 1 day in 51 patients(Table:3). Complications were minor and were seen in (Table:4).There was no intraoperative bleeding.There was no postoperative wound infection nor horner syndrome.Postoperative chest X-ray was performed only if the patient complained of breathing difficulty. Only 4 patients needed to have an X-ray and proved to had pneumothorax which necessitied chest tube insertion.

Postoperative care: Most patients were discharged home on the same day of the procedure and All patients were followed up for a minimum period of 6 months. Data were collected by review of medical charts, telephone and interviews. When asked for untoward side effects of thoracic endoscopic sympathectomy, 4patients $(7.1 \%)$ reported 
increased sweating elsewere on the body, ie, compensatory sweating, mainly on the trunk, abdomen, and thighs.Mostly, compensatory sweating was considered much less embarrassing than the original form of hyperhidrosis.No cases of gustatory sweating.18 cases complain from pleuritic chest pain,which improve with analgesic. The crucial question was whether patients were satisfied with the operative result.Despite the above-mentioned side effects, 51 of 56 patients $(91.1 \%)$ considered the result of endoscopie thoracic sympathectomy highly satisfying(table:5,6,7). Many of them even stated inexuberant terms that their life had completely changed into a much brighter and happier existence than before.

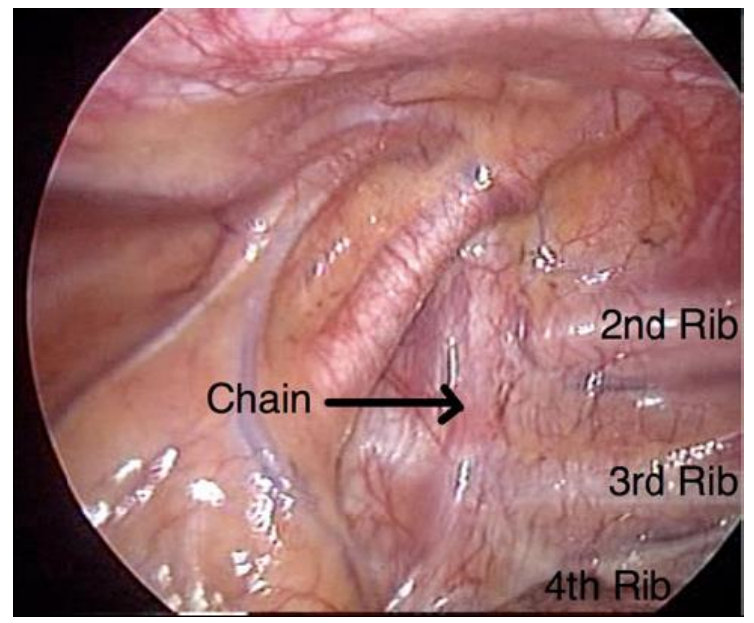

Figure(1): Identifying the chain

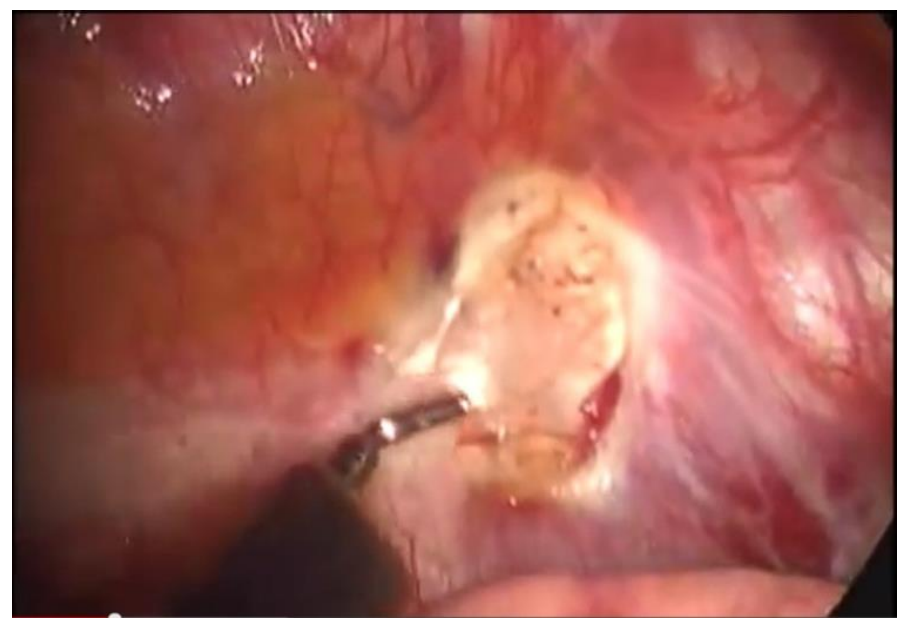

Figure (2): The transected ends of the sympathetic chain

Table (1): Gender distribution

\begin{tabular}{lllll}
\hline & & & Frequency & Percent \\
\hline \multirow{3}{*}{ Sex } & Female & $\mathbf{1 8}$ & $\mathbf{3 2 . 1}$ \\
\cline { 2 - 4 } & Male & $\mathbf{3 8}$ & $\mathbf{6 7 . 9}$ \\
\cline { 2 - 4 } & Total & $\mathbf{5 6}$ & $\mathbf{1 0 0 . 0}$ \\
\hline
\end{tabular}

Table (2): Age distribution among studied group

\begin{tabular}{ll}
\hline & Age \\
\hline $\mathbf{N}$ & 56 \\
\hline Mean \pm SD & $27.03 \pm 4.89$ \\
\hline Median (Range) & $26.0(19-34)$ \\
\hline
\end{tabular}

Table (3): Hospital stay

\begin{tabular}{llll}
\hline \multirow{2}{*}{ Hospital stay } & & $\mathrm{N}$ & $\%$ \\
& One day & $\mathbf{5 1}$ & $\mathbf{9 1 . 1}$ \\
\cline { 2 - 4 } & Two days & $\mathbf{5}$ & $\mathbf{8 . 9}$ \\
\hline Mean \pm SD & & $1.08 \pm 0.28$ & \\
\hline Median (Range) & & $1.0(1-2)$ & \\
\hline
\end{tabular}


Table (4): Postoperative Complications

\begin{tabular}{llll}
\hline \multirow{2}{*}{ Compensatory sweating } & & $\mathrm{N}$ & $\%$ \\
\hline \multirow{2}{*}{ Phest tube } & NO & $\mathbf{5 2}$ & $\mathbf{9 2 . 9}$ \\
\cline { 2 - 4 } & YES & $\mathbf{4}$ & $\mathbf{7 . 1}$ \\
& NO & $\mathbf{5 2}$ & $\mathbf{9 2 . 9}$ \\
\cline { 2 - 4 } & YES & $\mathbf{4}$ & $\mathbf{7 . 1}$ \\
\hline Intraoperative bleeding & NO & $\mathbf{5 2}$ & $\mathbf{9 2 . 9}$ \\
\cline { 2 - 4 } & YES & $\mathbf{4}$ & $\mathbf{7 . 1}$ \\
\hline Gustatory Sweating & NO & $\mathbf{5 6}$ & $\mathbf{1 0 0 . 0}$ \\
\cline { 2 - 4 } & YES & $\mathbf{0}$ & $\mathbf{0 . 0}$ \\
\hline Inter costal neuralgia & NO & $\mathbf{5 6}$ & $\mathbf{1 0 0 . 0}$ \\
\cline { 2 - 4 } & YES & $\mathbf{0 . 0}$ & $\mathbf{0 . 0}$ \\
\hline Pleuritic chest pain & NO & $\mathbf{3 7}$ & $\mathbf{6 6 . 1}$ \\
\cline { 2 - 4 } & YES & $\mathbf{1 9}$ & $\mathbf{3 3 . 9}$ \\
\hline Mortality & NO & $\mathbf{3 8}$ & $\mathbf{6 7 . 9}$ \\
\cline { 2 - 4 } & YES & $\mathbf{1 8}$ & $\mathbf{3 2 . 1}$ \\
\hline operative wound infection & NO & $\mathbf{5 6}$ & $\mathbf{1 0 0 . 0}$ \\
\cline { 2 - 4 } & YES & $\mathbf{0}$ & $\mathbf{0 . 0}$ \\
\hline Horner syndrome & NO & $\mathbf{5 6}$ & $\mathbf{1 0 0 . 0}$ \\
\cline { 2 - 4 } & YES & $\mathbf{0}$ & $\mathbf{0 . 0}$ \\
\hline
\end{tabular}

Table (5): Satisfaction

\begin{tabular}{llll}
\hline & & Frequenc & Percent \\
& & y & \\
\hline Satisfaction & Poor & $\mathbf{5}$ & $\mathbf{8 . 9}$ \\
\cline { 2 - 4 } & Good & $\mathbf{5 1}$ & $\mathbf{9 1 . 1}$ \\
\cline { 2 - 4 } & Total & $\mathbf{5 6}$ & $\mathbf{1 0 0 . 0}$ \\
\hline
\end{tabular}


Table (6): Association with outcome

\begin{tabular}{|c|c|c|c|c|c|c|c|}
\hline & & & Satisfact & & Total & $\mathrm{X}^{2}$ & $\mathrm{P}$ \\
\hline & & & POOR & YES & & & \\
\hline Sex & FEMALE & $\mathrm{N}$ & $\mathbf{0}$ & 18 & 18 & 2.6 & $\mathbf{0 . 1 0 7}$ \\
\hline & & $\%$ & $0.0 \%$ & $35.3 \%$ & $32.1 \%$ & & \\
\hline & MALE & $\mathrm{N}$ & 5 & 33 & 38 & & \\
\hline & & $\%$ & $100.0 \%$ & $64.7 \%$ & 67.9\% & & \\
\hline Compensatory sweating & NO & $\mathrm{N}$ & 4 & 48 & 52 & 1.36 & 0.24 \\
\hline & & $\%$ & $80.0 \%$ & $94.1 \%$ & $92.9 \%$ & & \\
\hline & YES & $\mathrm{N}$ & 1 & 3 & 4 & & \\
\hline & & $\%$ & $20.0 \%$ & $5.9 \%$ & $7.1 \%$ & & \\
\hline Chest tube & NO & $\mathrm{N}$ & 1 & 51 & 52 & 43.9 & $0.00 * *$ \\
\hline & & $\%$ & $20.0 \%$ & $100.0 \%$ & $92.9 \%$ & & \\
\hline & YES & $\mathrm{N}$ & 4 & $\mathbf{0}$ & 4 & & \\
\hline & & $\%$ & $80.0 \%$ & $\mathbf{0 . 0 \%}$ & $7.1 \%$ & & \\
\hline Pneumothorax & NO & $\mathrm{N}$ & 1 & 51 & 52 & 43.9 & $0.00 * *$ \\
\hline & & $\%$ & $20.0 \%$ & $100.0 \%$ & $92.9 \%$ & & \\
\hline & YES & $\mathrm{N}$ & 4 & $\mathbf{0}$ & 4 & & \\
\hline & & $\%$ & $80.0 \%$ & $0.0 \%$ & $7.1 \%$ & & \\
\hline Inter costal neuralgia & $\mathrm{NO}$ & $\mathrm{N}$ & 3 & 34 & 37 & 0.09 & 0.76 \\
\hline & & $\%$ & $60.0 \%$ & $66.7 \%$ & $66.1 \%$ & & \\
\hline & YES & $\mathrm{N}$ & 2 & 17 & 19 & & \\
\hline & & $\%$ & $40.0 \%$ & $33.3 \%$ & $33.9 \%$ & & \\
\hline Pleuritic chest pain & $\mathrm{NO}$ & $\mathrm{N}$ & 4 & 34 & 38 & 0.37 & 0.54 \\
\hline & & $\%$ & $80.0 \%$ & $66.7 \%$ & $67.9 \%$ & & \\
\hline & YES & $\mathrm{N}$ & 1 & 17 & 18 & & \\
\hline & & $\%$ & $20.0 \%$ & $33.3 \%$ & $32.1 \%$ & & \\
\hline Total & & $\mathrm{N}$ & 5 & 51 & 56 & & \\
\hline & & $\%$ & $100.0 \%$ & $100.0 \%$ & $100.0 \%$ & & \\
\hline
\end{tabular}

Chest tube and Pneumothorax sig associated with poor outcome

Table (7): Hospital stay and Satisfaction

\begin{tabular}{|c|c|c|c|c|c|c|c|}
\hline & & & Satisfact & & Total & $\mathrm{X}^{2}$ & $\mathrm{P}$ \\
\hline & & & POOR & YES & & & \\
\hline Hospital & 1 day & $\mathrm{N}$ & 0 & 51 & 51 & 56.0 & 0.00** \\
\hline stay & & $\%$ & $0.0 \%$ & $100.0 \%$ & $91.1 \%$ & & \\
\hline & 2 & $\mathrm{~N}$ & 5 & 0 & 5 & & \\
\hline & days & $\%$ & $100.0 \%$ & $0.0 \%$ & $8.9 \%$ & & \\
\hline Total & & $\mathrm{N}$ & 5 & 51 & 56 & & \\
\hline & & $\%$ & $100.0 \%$ & $100.0 \%$ & $100.0 \%$ & & \\
\hline
\end{tabular}

Two days Hospital stay sig associated with poor

\section{DISCUSSION}

Thoracoscopic sympathectomy is an apparently simple technique, although serious complications have been described, such as bleeding,Horner syndrome and pneumothorax ${ }^{(\mathbf{1 0})}$.Surgical resection of thoracic ganglia is considered sufficient for the cessation of palmar and axillary hyperhydrosis. Ganglionic resection distal to T2 ganglia decreases dramatically the incidence of Horner syndrome. Recently, sympathetic disconnection has been described using electrocautery, reportedly to avoid compensatory sweating ${ }^{(\mathbf{1 1})}$.

In recent years, numerous articles advocating different surgical techniques for 
accessing the thoracic sympathetic chain have been published. Those articles discuss indications, methods as ablation or resection (and its extent), complications, and more ${ }^{(\mathbf{1 1})}$.

This study conducted on fifty six patients, underwent bilateral thoracoscopic sympathectomy in the duration from Jul, 2012 to Jun, 2017 with Minimum 6 month follow up. All cases presented with bilateral primary palmar hyperhidrosis as a main complain which caused a grave professional and social handicap .

We consistently used two port technique with transection of sympathetic chain from $\mathrm{T} 2$ to $\mathrm{T} 4$ for all our patients. This agree with Wali, (12) who used two ports However,Krasna et al, ${ }^{(13)}$ used three ports for their patients.But Lardinois and Ris, ${ }^{(14)}$ reported the use of a single trans-axillary port with good results and a better cosmoses.

In our study the youngest patient was 19years old, The oldest was 34years old and the mean age was $27.03 \pm 4.89$ years. In a study done by Pedro et al ${ }^{(15)}$, which included 406 patients with age range from 13 to 66 years with an average age of $25.1 \pm 9.1$ years. However, the age was not significant in our study.

Male sex predominance in our study were 38 cases $(67.9 \%)$ while females were 18 cases $(32.1 \%)$. This ratio was $7: 3$, which was totally insignificant in our study but this is almost the reverse to most of the reports. Except for Lardinois and $\mathbf{R i s}^{(14)}$. from Switzerland who reported 1:1 ratio, Lin T, KUO S and Chou $\mathbf{M}^{(\mathbf{1 6})}$ reported 1:1.5 or $1: 3 \cdot 5 \cdot 1,4-7)$. Such as study done by Johannes et al $^{(17)}$, which included 178 patients while male 51 cases and female 127 cases. This may be related to the highly conservative society in our country, which makes women less exposed to society or discouraged from seeking medical treatment for this problem.

In our study, The hospital stay range from 1 to 2 days, From all patients 4 cases had postoperative hospital stay for 2 days, because they had prophylacted chest tube, 48 cases had postoperative hospital stay for one day, 4cases had postoperative developed pneumothorax and reqired to stay at hospital for 2 days for complete improvement. The mean hospital stay was $1.08 \pm 0.28$ day.

In a study done by Mark and Xiaolong in ${ }^{(\mathbf{1 8 )}}$. which included 121 patients, The hospital stay range from 1/2-3 day and The mean hospital stay was 1.2 day.

In a study done by Pedro et $\mathbf{a l}^{(15)}$ which included 406 patients, consisting of: 16 cases (3.9\%) developed post operative pneumothorax which required chest tube and need to stay at hospital for 2 days.

\section{CONCLUSION}

Video-assisted thoracoscopic bilateral sympathectomy is a rapid, reliable and safe technique. It presents important advantages over the staged procedures because it can be done in a single surgical procedure, requires placement of only two thoracic ports, avoiding the third port. The transaxillary bilateral approach provides as good if not better results and can certainly be done with similar or perhaps less morbidity. Furthermore, the bilateral approach reduces the time of patient recovery and reduces health-care costs.

Although the follow-up of the simultaneous bilateral operations in literature was significantly shorter than that of the staged procedures, the incidence of compensatory sweating is probably comparable since the overwhelming majority of compensatory sweating occurs very early following sympathectomy. In terms of recurrence of hyperhidrosis after sympathectomy, it has been shown that it occurs mainly during the first 2 years after operation. Therefore, to assess this issue appropriately, the follow-up should be long enough to permit the observation of recurrence.

Transection of sympathetic chain by electrocoagulation, namely sympathotomy, is claimed to have comparable results to that of resection of the chain. In addition it is simpler, faster with less incidence of compensatory sweating. Nevertheless, largescale prospective studies with extended follow-up periods are needed to confirm these results. 


\section{REFERENCES}

1-Roberto de Menezes Lyra. (2013): "Visual scale for the quantification of hyperhidrosis.". Bras Pneumol. 39 (4): 521-2.

2-Strutton DR, Kowalski JW, Glaser DA, Stang PE (2014): US prevalence of hyper-hidrosisand impact on individuals with axillary hyperhidrosis: Results from a national survey. J Am Acad Dermatol;51(2):241-8.

3-Jeganathan R, Jordan S and Jones M (2008): Bilateral thoracoscopic sympathectomy: results and long-term follow-up. Interact Cardiovasc Thorac Surg ; 7:67-70.

4-Prasad, A; Ali, M; Kaul, S (2010): "Endoscopic thoracic sympathectomy for primary palmar hyperidrosis". Surgi-cal endoscopy 24 (8): 1952-7. doi:10.1007/s00464-010-0885-5. PMID 20112111

5-Vary JC Jr (2015): "Selected Disorders of Skin Appendages-Acne, Alopecia, Hyperhidrosis". The Medical Clinics of North America. 99 (6): 11951211. doi:10.1016/j.mcna.2015.07.003. PMID 26476248

6-Roberto de Menezes Lyra, Campos JR, Kang DW, Loureiro Mde P, Furian MB, Costa MG, Coelho Mde S; Sociedade Brasileira de Cirurgia Torácica. (2014): "Guidelines for the prevention, diagnosis and treatment of compensatory hyperhidrosis.". J Bras Pneumol. 34 (11): 967-77. doi:10.1590/s1806-37132008001100013.PMID 19099105

7-Purtuloglu T, Atima A, Deniza S (2013): Effect of radiofrequency ablation and comparison with surgical sympathectomy in palmar hyperhidrosis. European Journal of Cardio-Thoracic Surgery; 43: e151-e154.

8- Franco C, Cajaraville J, Grimac F and Espan A (2011): Prospective study of percutaneous radiofrequency sympathicolysis in severe hyperhidrosis and facial blushing: efficacy and safety findings. European Journal of Cardiothoracic Surgery 40 (2011) 146-151.

9-Walles T, Somuncuoglu G, Steger V (2018): Longterm efficiency of endoscopic thoracic sympathicotomy: survey 10 years after surgery. Interact Cardio Vasc Thorac Surg 8:54-57.

10-Han PP, Kenny K, Dickman CA (2012): Thoracoscopic approaches to the thoracic spine: experience with 241 surgical procedures. Neurosurgery 51(Suppl 2):88-95

11-Naumann M, So Y, Argoff C, et al,(2016): Assessment: Botulinum neurotoxin in the treatment of autonomic disorders and pain (an evidence-based review), Report of the Therapeutics and Technology Assessment Subcommittee of the American Academy of Neurology. 70 pps 17071714.

12Wali M (2017) : Early Experience with Thoracoscopic Sympathectomy for Palmar Hyperhidrosis. Ann Thorac Cardiovasc Surg,. 9, ( 6 )Pp 351-354

13-Krasna K, Kawase H, Kawanishi M,( 2013) : Health-Related Quality of Life After Thoracoscopic Sympathectomy for Palmar Hyperhidrosis. Ann Thorac Surg ; 80: Pps 461-466.

14-Lardinois D, Ris H,(2018) : Minimally invasive video-endoscopic sympathectomy by use of a transaxillary single port approach. Eur J Cardiothorac Surg; 21: Pps 67-70.

15-Pedro M. Rodríguez, Jorge L et al, (2015) : Side effects, complications and outcome of thoracoscopic sympathectomy for palmar and axillary hyperhidrosis in 406 patients. European Journal of Cardio-Thoracic Surgery, 34, (3), Pps 514-519

16-Lin T, KUO S, Chou M,(2012): Uniportal Endoscopic Thoracic Sympathectomy For Treatment of Palmar and Axillary Hyperhidrosis: Analysis of 2000 Cases, J Neurosurgery ,51 (5),pps 84-87

17-Johannes S, Falk G, Peter A et al,(2016): Endoscopic Thoracic Sympathectomy for Severe Hyperhidrosis: Impact of Restrictive Denervation on Compensatory Sweating. The Annals of Thoracic Surgery. 81,( 3), Pps 1048-1055

18-Mark $K$ and Xiaolong J,(2010): Thoracoscopic Sympathectomy for for the treatment of a wide variety of disorders. European Journal of CardioThoracic Surgery,28,(2),Pp 415-419. 